\title{
Precision medicine needs an equity agenda
}

\author{
Enhancing population diversity in genetic databases and evaluating genetic scores in conjunction with other \\ disease factors will be needed to ensure a more equitable impact of precision medicine.
}

$\longrightarrow$ ince the release of the first draft of the human genetic sequence 20 years ago by The Human Genome Project international consortium, technical improvements in sequencing approaches and a staggering drop in the cost of genome sequencing have enabled an exponential increase in the number and size of genetic datasets. This flurry of genetic information has enabled better understanding of the biology of diseases and is opening up possibilities for applying this knowledge for more-precise clinical management of patients.

The identification of high-penetrance disease-causing mutations-such as germline pathogenic alterations in $B R C A 1 / 2$ that result in a predisposition to breast cancer, or APOE variants in Alzheimer's disease - and mutations that encode drivers of disease that are clinically actionable has made management of patients across most disease domains more precise. For certain complex traits, the clinical utility of polygenic risk scores for predicting the risk of disease is still under study. The direct-to-consumer genetic-testing company 23 andMe offers a predictor of polygenic risk scores for diabetes based on the analysis of a panel of markers, and, among other clinical studies underway, the WISDOM study aims to enroll 100,000 women to assess the added value of polygenic risk scores over standard mammograms for personalizing the lifetime risk of developing breast cancer and tailoring screening recommendations.

Although genetic scores seem to be getting closer to the clinic, the following longstanding concern among geneticists remains: that the lack of diversity in the data used to build those scores is likely to limit their impact in the real world. This means that the integration of polygenic risk scores for standardized risk assessment, although promising, may not result in better care across ethnic groups. A recent analysis of genome-wide association studies according to ancestry revealed that European-ancestry genomes account for the vast majority of reference genomes. Worryingly, researchers also found that this trend has not improved in recent years.

Historically, genetic studies spearheaded by researchers and consortia in the UK or the USA have included predominantly participants of European descent. Beyond the lack of knowledge on the genetic architecture of non-European people, it is of concern that medical decisions grounded on the analysis of Eurocentric genetic scores may amplify healthcare disparities. Pathogenic variants identified in European genomes might be completely irrelevant for non-European people and, similarly, putative disease-causing mutations in people of underrepresented groups may go undetected. Populations of African or Asian ancestries are widely underrepresented relative to the fraction of the world's population they represent, and other groups, such as Indigenous peoples in the USA, the Caribbean or Australia, have not been included in these efforts.

Aiming to overturn this tendency, several genetic initiatives seeking to expand the representation of populations that have been historically neglected in genetic research are underway. The H3Africa initiative, led by African scientists across 30 countries, is building genetic research capacity and improving the representation of African genomes in genomic databases, which, given their fundamental diversity and inter-individual variation, will expand the human catalog of disease-related genetic associations. Similarly, GenomeAsia 100K is building a map of genetic diversity across Asian populations, and the ongoing Polyethnic- 1000 project is characterizing cancer-predisposing genetic factors across various ethnic groups in the New York City area. Likewise, 23 andMe is diversifying their genetic databases to make their reference populations more ethnically diverse, aiming to expand the predictive accuracy of their tests beyond people of European ancestry.

Increasing the diversity in genetic studies will require that people from underrepresented groups are engaged early on and that they play strong leadership roles in these projects. Participants must be well informed and aware of the value that these studies may have for improving health in their communities. Mistrust of the use of their data, concerns about data privacy and ownership, and failure to consider their particular cultural habits have all historically hindered the participation of underrepresented groups in genetic studies.
On their end, researchers have started to explore ways to mend the errors of the past and reconnect with these communities. In the USA, the National Human Genome Research Institute has engaged with Alaska Native tribes-a population that suffers high rates of cardiovascular disease, diabetes and some types of tumors-to overcome logistical and communication barriers in the hope of encouraging their involvement in genetics research. In Australia, researchers are reflecting on lessons drawn from a genome-wide association study on rheumatic heart disease in Aboriginal Australian participants to improve consenting principles, data handling and communication, and to pave the way to future partnerships with these communities in genetic studies.

Beyond expanding the diversity of genetic databases, the clinical implementation of genetic scores faces additional hurdles. Assays for understanding the functional relevance of genetic variants need to be developed, and the interaction of disease-related genetic variants with socio-economic and environmental factors needs to be better understood to determine the relative contribution of genetic risks. Toward implementation, multi-disciplinary medical teams need to develop a culture that incorporates genetic indicators in routine decision-making so the value of clinical genetics in precision medicine can be better ascertained. Finally, regulatory agencies and health systems need to closely monitor the robustness and impact of genetic tests to ensure that these do not perpetuate disparities or have a detrimental impact on people's health.

Twenty years after release of the first human genome, genomic profiling is becoming a tool in mainstream precision medicine across health conditions. Realizing its full potential, however, will require gaining a more diverse perspective of genetic variability across human populations, and their diversity within, to ensure that the clinical application of genetics is equitable and that it reaches global impact in the next twenty years.

Published online: 14 May 2021 https://doi.org/10.1038/s41591-021-01373-y 